Math. Model. Nat. Phenom.

Vol. 5, No. 7, 2010, pp. 97-102

DOI: $10.1051 / \mathrm{mmnp} / 20105716$

\title{
A Global Stochastic Optimization Method for Large Scale Problems
}

\author{
W. El Alem ${ }^{1,2 *}$, A. El Hami ${ }^{2}$, R. Ellaia ${ }^{1}$ \\ ${ }^{1}$ Laboratory of study and research in applied mathematics, Mohammed V University \\ EMI, BP. 765, Ibn Sina avenue, Agdal, Rabat, Morocco \\ ${ }^{2}$ Laboratory of mechanics of Rouen, national institute for applied sciences, Rouen \\ BP 08, university avenue 76801, St Etienne du Rouvray Cedex, France
}

\begin{abstract}
In this paper, a new hybrid simulated annealing algorithm for constrained global optimization is proposed. We have developed a stochastic algorithm called ASAPSPSA that uses Adaptive Simulated Annealing algorithm (ASA). ASA is a series of modifications to the basic simulated annealing algorithm (SA) that gives the region containing the global solution of an objective function. In addition, Simultaneous Perturbation Stochastic Approximation (SPSA) method, for solving unconstrained optimization problems, is used to refine the solution. We also propose Penalty SPSA (PSPSA) for solving constrained optimization problems. The constraints are handled using exterior point penalty functions. The combination of both techniques ASA and PSPSA provides a powerful hybrid optimization method. The proposed method has a good balance between exploration and exploitation with very fast computation speed, its performance as a viable large scale optimization method is demonstrated by testing it on a number of benchmark functions with 2 - 500 dimensions. In addition, applicability of the algorithm on structural design was tested and successful results were obtained.
\end{abstract}

Key words: global optimization, structural optimization, simultaneous perturbation stochastic approximation

AMS subject classification: 49Q10, 90C90, 78M50, 90C26, 74P05

${ }^{*}$ Corresponding author. E-mail: welalem@yahoo.fr 


\section{Introduction}

In the present paper we focus on the improvement of the efficiency of structural optimization. In typical structural optimization problems there may be many locally minimum configurations. For that reason, the application of a global method remains essential. Global optimization should take part in structural problems. Structural engineering and mathematical programming techniques should, both of them, collaborate in some way to develop a powerful and sophisticated programming system for structural optimization. The present paper focuses on the enhancement of simulated annealing algorithm by proposing the new hybrid method ASAPSPSA. The proposed method was designed in order to find the absolute minimum of an objective function without being sensitive to the starting point. It is capable of handling problems subject to any number of design variables or equality/inequality constraints, the solution is reached so that a reasonable compromise is made between the global solution and a shorter computational time. Numerical results demonstrate the applicability, accuracy and efficiency of the suggested method for structural optimization.

\section{The proposed method}

SA has been developed rapidly since the past 20 years as an effective and simple optimization technique. The superiority of SA lies in its good robustness and convenience to realize global optimization. The basic simulated annealing algorithm used in this paper is a simulated annealing based on the work of Van Laarhoven and Aarts [9], the algorithm is well described by Sitarz [6]. Although, simulated annealing is a global optimization method, it suffers from some disadvantages such as insufficient accuracy or slow convergence to the global minimum. In order to overcome these shortcomings we propose two modifications viz, (a) a new stopping rule and (b) an application of a local search method SPSA based on the work of J. Spall ([7], [8]). Since SPSA is an unconstrained optimization method, we have applied the exterior penalty method for further details see [5] to penalize constraints. We call it Penalty Simultaneous Perturbation Stochastic Approximation (PSPSA) method. The hybridization of these two methods allows us to design the new method ASAPSPSA for constrained optimization problems. The ASAPSPSA algorithm is as follows:

\section{Algorithm 1. ASAPSPSA Algorithm}

Step 1: Initialization and coefficient selection.

Initialize the starting point at random and the initial temperature $T$.

Step 2: Search the region containing the global solution.

Use ASA to reach the region containing the global solution according to the stopping role. 
Step 3: search the global solution.

Keep the solution founded by ASA and restart the search with PSPSA method.

Step 4: Iteration or termination.

Terminate the algorithm if there is little change in several successive iterations or the maximum number of iterations has been reached.

\section{Numerical results}

\subsection{Benchmark test functions}

Several benchmark functions, see Appendix, have been performed. Basic information about the benchmark functions are reported in Table 1, global optimization methods used for performance analysis are provided by Table 2 and computational results are summarized in Table 3 for each function.

\begin{tabular}{llllll}
\hline Function (Name) & Search space & $\begin{array}{l}\text { Number } \\
\text { of local } \\
\text { minima }\end{array}$ & The global optimum & $f_{\text {min }}$ & reference \\
\hline$D J$ (De Jong) & {$[-100,100]^{d}$} & 1 & $(0,0, \ldots ., 0)$ & 0 & {$[10]$} \\
$C A$ (Camel) & {$[-10,10]^{d}$} & 6 & $( \pm 0.089842, \mp 0.712656)$ & -1.031628 & {$[10],[4]$} \\
$S H U$ (Shubert) & {$[-10,10]^{d}$} & 760 & $d .3^{d}(-1.42513,-0.80032)$ & -186.730909 & {$[4]$} \\
\hline
\end{tabular}

Table 1: Basic information about 3 Benchmark functions, $d$ is the dimension of the test functions

\begin{tabular}{|c|c|c|}
\hline Method & Name & Reference \\
\hline$e P S O$ & extrapolation Particle Swarm Optimization & {$[1]$} \\
\hline$N F F$ & New Filled Function & {$[11]$} \\
\hline$G L P_{\tau} S$ & $\begin{array}{l}\text { Genetic algorithms Low-discrepancy sequences of } \\
\text { Points and Simplex search }\end{array}$ & [2] \\
\hline$G R S A$ & Gradient Simulated Annealing & {$[12]$} \\
\hline$O D E$ & Orthogonal based Differential Evolution & {$[3]$} \\
\hline$A S A P S P S A$ & Adaptive Simulated Annealing Penalty SPSA & This paper \\
\hline
\end{tabular}

Table 2: Global optimization methods used for performance analysis

The performance of the proposed new algorithm is examined in literature $[2,4,10]$ on 3 benchmark functions. Table 3 summarizes the performance of the proposed method and compares the 


\begin{tabular}{llll}
\hline Test functions & $\mathrm{d}$ & $A S A P S P S A$ & Other methods \\
\hline & 30 & $2.6686 \mathrm{e}^{-61}$ & $O D E: 2.06 \mathrm{e}^{-23}$ \\
& 100 & $5.6808 \mathrm{e}^{-59}$ & $G L P_{\tau} S: 5 \mathrm{e}^{-03}$ \\
$D J$ & 150 & $8.5623 \mathrm{e}^{-59}$ & $G L P_{\tau} S: 3.8 \mathrm{e}^{-03}$ \\
& 500 & $1.8059 \mathrm{e}^{-56}$ & $G R S A: 0.98$ \\
$S H U$ & 2 & -186.730908831023 & $N F F:-186.730908830971$ \\
$C A$ & 2 & -1.03162841 & $e P S O:-1.031604$ \\
\hline
\end{tabular}

Table 3: Performance of $A S A P S P S A$ on 3 test functions, $\mathrm{d}$ is the dimension of the test functions.

results found with other methods. To evaluate the efficiency of the new method, a high dimensional function used in papers $[2,10]$ is selected to test $A S A P S P S A$ and comparison with the methods $O D E, G L P_{\tau} S$ and $G R S A[2,3,12]$ is carried out. As shown in Table 3, the ASAPSPSA can converge fairly close to the global optimum. When compared with other methods $A S A P S P S A$ demonstrate its accuracy in achieving the global solution efficiently.

\subsection{Structural optimization}

The structural problem considered in this work deals with a hexagonal steel plate, using thickness $T_{1}$ and fillet radius FILL as the optimization parameters ( see Figure 2). This problem uses a $2-D$ model and takes advantage of symmetry. The loading is tensile pressure (traction) of $50 \mathrm{MPa}$ at the three flat faces. The purpose of this optimization problem is to minimize the volume $\mathrm{V}$ of the triangular plate without exceeding the allowable stress $\sigma_{v o n}$. The maximum stress anywhere in the triangular plate should not exceed $150 \mathrm{MPa}$.

\begin{tabular}{lcc}
\hline & Initial point & ASAPSPSA \\
\cline { 2 - 3 } Volume & 22967.2 & 17952 \\
$\sigma_{\text {von }}$ & 56.727 & 149.8627 \\
$T_{1}$ & 30 & 20.0001 \\
FIL & 10 & 7.3160 \\
\hline
\end{tabular}

Table 4: Numerical results

The experimental results show that the present method has proved the robustness and high performance of its algorithm. Calculations show that ASAPSPSA achieves the optimal solution (see Table 4). The optimal volume reduction is $24.29 \%$ from initial shape (see Fig 2 and 3), witch leads to economic structure. 


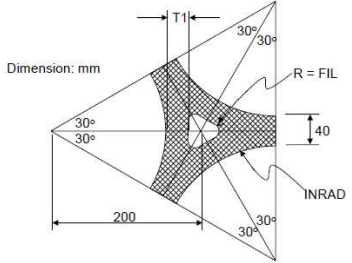

Figure 1: Triplate



Figure 2: Triplate with initial Figure 3: Optimal triplate using point

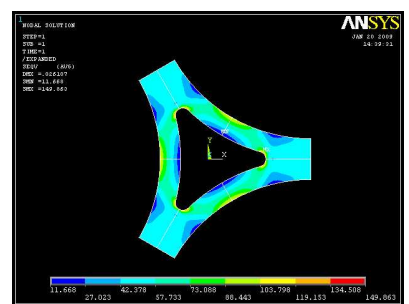

ASAPSPSA method

\section{Conclusion}

In this paper a new global hybrid method ASAPSPSA for constrained optimization problems has been proposed. The new algorithm can be widely applied to a class of global optimization problems for continuous functions. The experimental results show that the present method has proved the robustness and high performance of its algorithm. We can clearly see from the numerical results that the algorithm can yield the global optimum with high accuracy.

\section{Acknowledgements}

This work was supported by CMIFM, A.I. EGIDE, number: MA/07/173.

\section{Appendix}

$D J=\sum_{i=1}^{n} x_{i}^{2}$

$S H U=\prod_{i=1}^{n} \sum_{j=1}^{5} j \cos \left[(j+1) x_{i}+j\right]$

$C A=4 x_{1}^{2}-2.1 x_{1}^{4}+\frac{1}{3} x_{1}^{6}+x_{1} x_{2}-4 x_{2}^{2}+4 x_{2}^{4}$

\section{References}

[1] M. S. Arumugam, M. V. C. Rao, A. W. C. Tan. A novel and effective particle swarm optimization like algorithm with extrapolation technique. Applied Soft Computing, 9 (2009), No. 1, 308-320.

[2] A. Georgieva, I. Jordanov. Global optimization based on novel heuristics, low-discrepancy sequences and genetic algorithms. European Journal of Operational Research, 196 (2009), 413-422. 
[3] W. Gong, Z. Cai, L. Jiang. Enhancing the performance of differential evolution using orthogonal design method. Applied Mathematics and Computation, 206 (2008), No. 1, 56-69.

[4] Z. Huang, X. Miao, P. Wang. A revised cut-peak function method for box constrained continuous global optimization. Applied Mathematics and Computation 194 (2007), No. 1, 224-233.

[5] D. G. Luenberger. Introduction to linear and nonlinear programming. Addison Wesley, 1973.

[6] S. Sitarz. Ant algorithms and simulated annealing for multicriteria dynamic programming. Computers and Operations Research, 36 (2009), No. 2, 433-441.

[7] J. C. Spall. Multivariate stochastic approximation using a simultaneous perturbation gradient approximation. IEEE Transactions on Automatic Control, 37 (1992), No. 3, 332-341.

[8] J. C. Spall. Adaptive stochastic approximation by the simultaneous perturbation method. IEEE Transactions on Automatic Control, 45 (2000), No. 10, 1839-1853.

[9] PJM. Van Laarhoven, EHL. Aarts. Simulated annealing: theory and applications. Dordrecht: D. Reidel Publishing Company, Kluwer, 1987.

[10] L. Wang, K. Chen, Y. S. Ong (Eds). Advances in Natural Computation. Part III, Springer Science \& Business Publisher, Changsha, China, 2005.

[11] C. Wang, Y. Yang, J. Li. A new filled function method for unconstrained global optimization. Journal of Computational and Applied Mathematics, 225 (2009), No. 1, 68-79.

[12] Y. J. Wang., J. S. Zhang. An efficient algorithm for large scale global optimization of continuous functions. Journal of Computational and Applied Mathematics, 206 (2007), No. 2, 1015-1026. 